\title{
PHOTOEXCITATION OF POLARIZED ATOMS BY POLARIZED RADIATION
}

\author{
A. Kupliauskienè \\ Vilnius University Research Institute of Theoretical Physics and Astronomy, A. Goštauto 12, LT-01108 Vilnius, Lithuania \\ E-mail: akupl@itpa.lt
}

Received 1 December 2003

\begin{abstract}
An expression for the probability of the excitation of polarized atoms by polarized radiation is obtained by using the graphical technique of angular momentum. In the case of pure states, the applied method is an alternative to that of density matrix formalism. The obtained expression enables one to describe the polarization state of an excited atom and can be used to derive formulas for the special cases under specific experimental conditions as well as for the photoexcitation as the first step process. The application of obtained expressions for the description of multistep processes is discussed. The photoexcitation of unpolarized atoms by polarized radiation and polarized atoms by unpolarized radiation is considered as the examples of practical application of the obtained general expression.
\end{abstract}

Keywords: excited atoms, polarization, photoexcitation

PACS: 31.50.Df, 29.25.Pj

\section{Introduction}

The laser [1] and tunable synchrotron radiation allows one not only to ionize an electron of a specific outer or inner shell of an atom but also to excite it to a specific orbital $[2,3]$. The state of the produced atom is polarized if the radiation is polarized. Thus, the excitation of atoms by polarized radiation is one of the ways to produce atoms in polarized states for further measurements $[4,5]$. In the case of inner-shell ionization, the ions can emit the fluorescence radiation $[6,7]$ that provides information about the polarization state of an atom before the ionization. The decay of atomic states via the emission of a photon is the only way when electrons are excited from outer shell. The emission of an electron is another way of decay of an excited atomic state in the case of the inner-shell ionization [8]. Thus, the photoexcitation can be used as a first step to create a resonant state with well-defined angular momentum and parity for further investigations of polarization and angular correlation phenomena $[8,9]$.

The main aim of the present work was the derivation of a general expression for the probability of the excitation of polarized atoms with polarized radiation and the application of this expression for specific cases to describe the polarization used in measurements. The application of the general formula for description of the photoexcitation as the first step process is also considered. For derivation of the expression for excitation probability, the graphical technique of angular momentum [10] is applied. So far, the investigations of the photoexcitation of polarized atoms was performed only by using the methods of density matrix $[11,12]$, therefore, our treatment is an alternative to the density matrix formalism. Some improvements of the graphical technique and methods of the ordinary atomic theory suggested in [13-15] have also found an application in the present work.

\section{Derivation of the photoexcitation probability}

To obtain the general expression for the excitation of polarized atoms by a polarized radiation the following process was studied:

$$
A\left(\alpha_{0} J_{0} M_{0}\right)+h \nu\left(\widehat{\epsilon}_{\lambda} \mathbf{k}_{0}\right) \rightarrow A^{*}\left(\alpha_{1} J_{1} M_{1}\right) .
$$

Here an atom $A$ in the state $\alpha_{0} J_{0} M_{0}$ is excited by the electromagnetic radiation into the state $\alpha_{1} J_{1} M_{1}$, where $\alpha$ defines the configuration and other quantum numbers, $J$ is total angular momentum, and $M$ is its projection. The electromagnetic radiation is described by the wave vector $\mathbf{k}_{0}$ and unit vector $\widehat{\epsilon}_{\lambda}$ of the polarization ( $\lambda$ is the helicity, $\lambda= \pm 1$ ). The system of atomic units is used in the present work $(\hbar=e=m=1$, 
$c=137$, unless these constants are displayed explicitly). It is assumed that the fine structure splitting $\gg$ line width $\gg$ hyperfine structure splitting. Then the states of an atom can be characterized by the total angular momentum of all electronic shells. The modifications enabling the calculations of probability in a case when hyperfine structure is important will be described below. In the present work, it is assumed that the directions for the measurement of the projections $M_{0}$ and $M_{1}$ may be different.

The differential cross-section of the process (1) can be written as follows [12]:

$$
\begin{aligned}
& \frac{\mathrm{d} \sigma\left(\alpha_{0} J_{0} M_{0} \widehat{\epsilon}_{\lambda} \mathbf{k}_{0} \rightarrow \alpha_{1} J_{1} M_{1}\right)}{\mathrm{d} \Omega} \\
& =2 \pi^{2}\left[\int\left\langle\alpha_{1} J_{1} M_{1}|\mathbf{J}(\mathbf{r})| \alpha_{0} J_{0} M_{0}\right\rangle \mathbf{A}_{\lambda \mathbf{k}_{0}}(\mathbf{r}) \mathrm{d} \mathbf{r}\right] \\
& \quad \times\left[\int\left\langle\alpha_{1} J_{1} M_{1}|\mathbf{J}(\mathbf{r})| \alpha_{0} J_{0} M_{0}\right\rangle \mathbf{A}_{\lambda \mathbf{k}_{0}}(\mathbf{r}) \mathrm{d} \mathbf{r}\right]^{*} .
\end{aligned}
$$

Here $\mathbf{J}(\mathbf{r})$ stands for the operator of the current of electrons and $\mathbf{A}_{\lambda \mathbf{k}_{0}}(\mathbf{r})$ is the operator of the vector potential of electromagnetic field. Taking into account that $k r \gg 1$, assuming an arbitrary direction of the incidence $\mathbf{k}_{0}$ of the photon, and inserting the multipole expansion for the $\mathbf{A}_{\lambda \mathbf{k}_{0}}(\mathbf{r})$, the expression in the right square brackets of (2) acquires the form [12]

$$
\begin{aligned}
\int\left\langle\alpha_{1} J_{1} M_{1}|\mathbf{J}(\mathbf{r})| \alpha_{0} J_{0} M_{0}\right\rangle \mathbf{A}_{\lambda k_{0}}(\mathbf{r}) \mathrm{d} \mathbf{r} \\
=\sum_{p=0,1} \sum_{k=1}^{\infty} \sum_{q=-k}^{k} \mathrm{i}^{k}(-\mathrm{i} \lambda)^{p}\left[\frac{k+1}{k}\right]^{1 / 2} \frac{k_{0}^{k-1 / 2}}{(2 k-1) ! !} \\
\quad \times D_{q \lambda}^{k}\left(\widehat{k}_{0}\right)\left\langle\alpha_{1} J_{1} M_{1}\left|\mathcal{Q}_{k q}^{p}\right| \alpha_{0} J_{0} M_{0}\right\rangle \\
=\sum_{k=1}^{\infty} \sum_{q=-k}^{k}\left\langle\alpha_{1} J_{1} M_{1}\left|Q_{q}^{(k)}\right| \alpha_{0} J_{0} M_{0}\right\rangle D_{q \lambda}^{k}\left(\widehat{k}_{0}\right), \\
\left\langle\alpha_{1} J_{1} M_{1}\left|Q_{q}^{(k)}\right| \alpha_{0} J_{0} M_{0}\right\rangle \\
=k_{0}^{k-1 / 2} \sum_{p=0,1}\left[\frac{k+1}{k}\right]^{1 / 2} \frac{\mathrm{i}^{k}(-\mathrm{i} \lambda)^{p}}{(2 k-1) ! !} \\
\quad \times\left\langle\alpha_{1} J_{1} M_{1}\left|\mathcal{Q}_{k q}^{p}\right| \alpha_{0} J_{0} M_{0}\right\rangle .
\end{aligned}
$$

Here $D_{q \lambda}^{k}\left(\widehat{k}_{0}\right)$ is the Wigner rotation matrix [16], and $p=0$ indicates the operator of electric multipole transition $(\mathrm{E} k)$

$$
\begin{aligned}
& \left\langle\alpha_{1} J_{1} M_{1}\left|\mathcal{Q}_{k q}^{0}\right| \alpha_{0} J_{0} M_{0}\right\rangle \\
& =\left[\frac{4 \pi}{2 k+1}\right]^{1 / 2} \sum_{j}\left\langle\alpha_{1} J_{1} M_{1}\left|r_{j}^{k} Y_{k q}(\widehat{r})\right| \alpha_{0} J_{0} M_{0}\right\rangle,
\end{aligned}
$$

and $p=1$ is for the magnetic multipole transition $(\mathrm{M} k)$

$$
\begin{aligned}
\left\langle\alpha_{1} J_{1} M_{1}\left|\mathcal{Q}_{k q}^{1}\right| \alpha_{0} J_{0} M_{0}\right\rangle \\
=\left[\frac{4 \pi}{2 k+1}\right]^{1 / 2} \sum_{j}\left\langle\alpha_{1} J_{1} M_{1}\right| \nabla r_{j}^{k} Y_{k q}(\widehat{r}) \\
\quad \times\left[\frac{l_{j}}{k+1}+s_{j}\right]\left|\alpha_{0} J_{0} M_{0}\right\rangle .
\end{aligned}
$$

Note that the parities of the magnetic and electric multipole fields are $(-1)^{k}$ and $(-1)^{k+1}$, respectively. Only the magnetic $(\mathrm{M} k)$ and electric $(\mathrm{E} k)$ part contributes to transitions between specific electronic states owing to parity selection rules. Since we are considering pure photon states, there is no need to introduce Stokes parameters explicitly. In electrical dipole approximation, the matrix element (4) is as follows:

$$
\begin{aligned}
& \left\langle\alpha_{1} J_{1} M_{1}\left|Q_{q}^{1}\right| \alpha_{0} J_{0} M_{0}\right\rangle \\
& \quad=\sqrt{2 k_{0}}\left\langle\alpha_{1} J_{1} M_{1}\left|\mathcal{Q}_{1 q}^{0}\right| \alpha_{0} J_{0} M_{0}\right\rangle .
\end{aligned}
$$

The helicity $\lambda= \pm 1$ describes the right-hand and left-hand circular radiation. In the case of any polarization $\epsilon$, the polarization may be expressed via the circular polarizations. Then

$$
\mathbf{A}_{\epsilon \mathbf{k}_{0}}(\mathbf{r})=\alpha \mathbf{A}_{\lambda=+1 \mathbf{k}_{0}}(\mathbf{r})+\beta \mathbf{A}_{\lambda=-1 \mathbf{k}_{0}}(\mathbf{r})
$$

has to be inserted into Eq. (3) to obtain the expression for the excitation cross-section (2).

Since there is no dependence on the direction of $\mathbf{k}_{0}$ in the electrical dipole approximation, the calculated angular distributions of the emitted radiation are symmetric with respect to $\mathbf{k}_{0}$ reversal. Retaining terms up to the second order, the calculation then includes electric dipole - electric quadrupole (E1-E2) and electric dipole - magnetic dipole (E1-M1) cross terms, i.e. interference between the E1 photoionization amplitudes and those for E2 and M1 interactions, but excludes E1-M2, M1-M1, and E2-M1 
cross terms that are of the order $\left(k_{0} r\right)^{2}$. The E1-E2 and E1-M1 cross terms have odd parity and produce calculated asymmetry in angular distributions of radiation with respect to the reversal of the direction of $\mathbf{k}_{0}$ [17].

Sometimes it is more convenient to analyse the polarization state of a particle with respect to the direction that differs from the one used for the calculation of the matrix element, where all particles of the process (1) should be described in the same coordinate system, and their projections of the angular momentum are on the same quantization axis. For switching from the wave function $|J M\rangle$ defined in the laboratory fixed direction to the wave function $|J \widetilde{M}\rangle$ of the atomic frame, the coordinate rotation transformation [16] is used:

$$
|J M\rangle=\sum_{\widetilde{M}} D_{\widetilde{M}, M}^{J}(\widehat{J})|J \widetilde{M}\rangle .
$$

Here $D_{\widetilde{M}, M}^{J}(\widehat{J})$ denotes the Wigner rotation matrix [16], and the hat on $J$ indicates the rotation by solid angle that transforms the atomic frame into the laboratory one used for the polarization measurements of the characteristics depending on $J$. In the laboratory system of coordinates the matrix element $\left\langle\alpha_{1} J_{1} M_{1}\left|Q_{q}^{(k)}\right| \alpha_{0} J_{0} M_{0}\right\rangle$ can be written, by taking into account Eq. (9), in the form

$$
\begin{aligned}
& \left\langle\alpha_{1} J_{1} M_{1}\left|Q_{q}^{(k)}\right| \alpha_{0} J_{0} M_{0}\right\rangle \\
& =\sum_{\widetilde{M}_{0}, \widetilde{q}^{,} \widetilde{M}_{1}}\left\langle\alpha_{1} J_{1} \widetilde{M}_{1}\left|Q_{\widetilde{q}}^{(k)}\right| \alpha_{0} J_{0} \widetilde{M}_{0}\right\rangle \\
& \quad \times D_{\widetilde{M}_{0} M_{0}}^{J_{0}}\left(\widehat{J}_{0}\right) D_{\widetilde{q} q}^{k}\left(\widehat{k}_{0}\right) D_{\widetilde{M}_{1} M_{1}}^{* J_{1}}\left(\widehat{J}_{1}\right) .
\end{aligned}
$$

The matrix element $\left\langle\alpha_{1} J_{1} \widetilde{M}_{1}\left|Q_{\widetilde{q}}^{(k)}\right| \alpha_{0} J_{0} \widetilde{M}_{0}\right\rangle$ is defined in the atomic frame. The angular momentum part of this matrix element is represented by diagram $A_{1}$ in Fig. 1 [10], where the open lines are defined in the laboratory fixed coordinate system, the circles with D inside stand for the Wigner rotation matrices (9), and the rectangle at the lines representing the total angular quanta $J$ of the initial and final states stands for the configuration and other quantum numbers. The application of the Wigner-Eckart theorem [10] enables us to separate the part that is independent of the space rotation (diagram $\mathrm{A}_{2}$ in Fig. 1) and a Clebsch-Gordan coefficient (diagram $A_{3}$ in Fig. 1) that, together with the Wigner rotation ma- trices (see Fig. 1), describes the directions of an atom in the initial and final states and a photon. The second Clebsch-Gordan coefficient comes up from the diagram of the complex conjugate matrix element (2).

For further simplification of the part describing the space rotation dependence, the following relation can be used:

$$
\begin{aligned}
& D_{\widetilde{M} M}^{J}(\widehat{J}) D_{\widetilde{M}^{\prime} M}^{* J^{\prime}}(\widehat{J}) \\
& \quad=\sum_{K, N} T_{N}^{* K}\left(J, J^{\prime}, M \mid \widehat{J}\right)\left[\begin{array}{ccc}
J^{\prime} & K & J \\
\widetilde{M}^{\prime} & N & \widetilde{M}
\end{array}\right] .
\end{aligned}
$$

The tensor in Eq. (11) is defined as [15]

$$
\begin{aligned}
& T_{N}^{* K}\left(J, J^{\prime}, M \mid \widehat{J}\right) \\
& \quad=(-1)^{J^{\prime}-M}\left[\frac{4 \pi}{2 J+1}\right]^{1 / 2}\left[\begin{array}{ccc}
J & J^{\prime} & K \\
M & -M & 0
\end{array}\right] Y_{K N}^{*}(\theta, \phi) .
\end{aligned}
$$

Three Clebsch-Gordan coefficients for each momentum $J_{0}, J_{1}$, and $k$ (see Eq. (11)) are used to perform the summation over the projections $\widetilde{M}_{0}, \widetilde{M}_{0}^{\prime}$, $\widetilde{M}_{1}, \widetilde{M}_{1}^{\prime}, \widetilde{q}, \widetilde{q}^{\prime}$ in Eq. (10) and its complex conjugate. All Clebsch-Gordan coefficients coming up from diagram $\mathrm{A}_{1}$ and its complex conjugate counterpart as well as for each angular momentum from Eq. (11) are shown in diagram $\mathrm{A}_{4}$. This summation was performed graphically, and the result is represented by diagram $A_{5}$. The closing of open lines from $A_{5}$ produces the diagram $\mathrm{A}_{6}$ invariant under space rotation and the diagram $\mathrm{A}_{7}$ describing rotation properties of the excitation cross-section (2). The bow on the open lines of diagram $A_{7}$ represents the operator (11). The final expression for the excitation probability (2) can be written by using diagrams $\mathrm{A}_{2}, \mathrm{~A}_{6}$, and $\mathrm{A}_{7}$ in the following form:

$$
\begin{aligned}
& \frac{\mathrm{d} \sigma\left(\alpha_{0} J_{0} M_{0} \hat{\epsilon} \mathbf{k}_{0} \rightarrow \alpha_{1} J_{1} M_{1}\right)}{\mathrm{d} \Omega} \\
& =C \sum_{K_{0}, K_{r}, K_{1}, k \cdot k^{\prime}} \frac{1}{2 K_{1}+1} B^{r}\left(K_{0}, K_{r}, K_{1}, k, k^{\prime}\right) \\
& \times \sum_{N_{0}, N_{r}, N_{1}, q}\left[\begin{array}{lll}
K_{0} & K_{r} & K_{1} \\
N_{0} & N_{r} & N_{1}
\end{array}\right] T_{N_{0}}^{* K_{0}}\left(J_{0}, J_{0}, M_{0} \mid \widehat{J}_{0}\right) \\
& \times T_{N_{r}}^{* K_{r}}\left(k, k^{\prime}, q \mid \widehat{k}_{0}\right) T_{N_{1}}^{K_{1}}\left(J_{1}, J_{1}, M_{1} \mid \widehat{J}_{1}\right),
\end{aligned}
$$



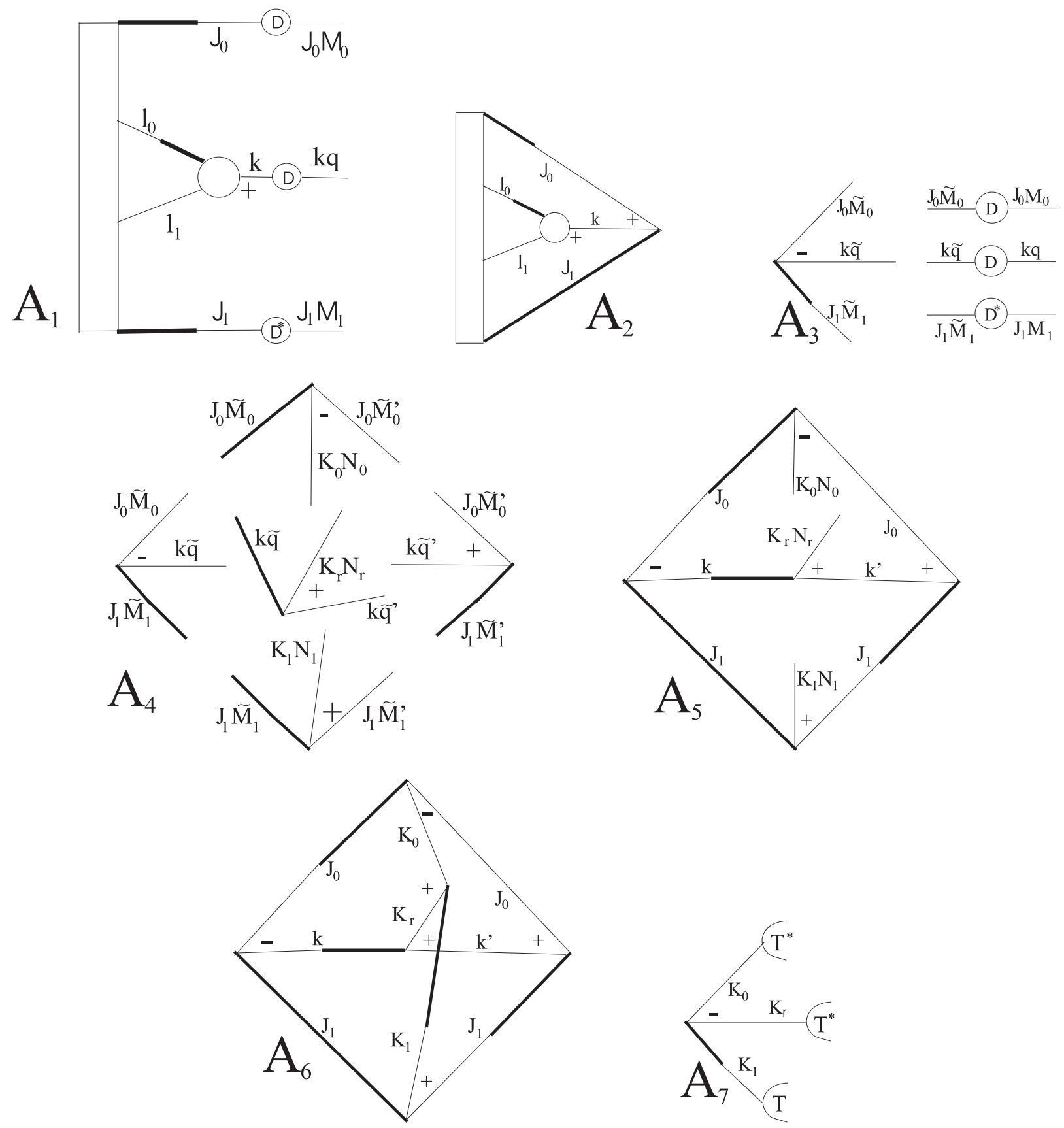

Fig. 1. The diagrams of angular momentum theory used to derive the expression for the probability of excitation of polarized atoms by polarized radiation.

where

$$
\begin{aligned}
B^{r}( & \left.K_{0}, K_{r}, K_{1}, k . k^{\prime}\right) \\
= & \left(\alpha_{1} J_{1}\left\|Q^{(k)}\right\| \alpha_{0} J_{0}\right)\left(\alpha_{1} J_{1}\left\|Q^{\left(k^{\prime}\right)}\right\| \alpha_{0} J_{0}\right)^{*} \\
& \times\left\{\begin{array}{ccc}
J_{0} & K_{0} & J_{0} \\
k & K_{r} & k^{\prime} \\
J_{1} & K_{1} & J_{1}
\end{array}\right\}\left[\left(2 J_{0}+1\right)\left(2 J_{1}+1\right)\right. \\
& \left.\times(2 k+1)\left(2 K_{1}+1\right)\right]^{1 / 2} .
\end{aligned}
$$

In Eq. (14) the relation

$$
\left(\alpha_{1} J_{1}\left\|Q^{(k)}\right\| \alpha_{0} J_{0}\right)=\left[2 J_{1}+1\right]^{1 / 2}\left\langle\alpha_{1} J_{1}\left\|Q^{(k)}\right\| \alpha_{0} J_{0}\right\rangle
$$

is taken into account. In Eq. (13), $C=2 \pi^{2}$.

In the case when hyperfine structure is important, the reduced matrix element $\left(\alpha_{1} J_{1}\left\|Q^{(k)}\right\| \alpha_{0} J_{0}\right)$ in Eq. (14) should be changed by $\left(\alpha_{1} J_{1}(I) F_{1}\left\|Q^{(k)}\right\| \alpha_{0} J_{0}(I) F_{0}\right)$. A simple relation between these two matrix elements holds: 


$$
\begin{aligned}
\left(\alpha_{1} J_{1}(I) F_{1}\left\|Q^{(k)}\right\| \alpha_{0} J_{0}(I) F_{0}\right) \\
=(-1)^{F_{0}-J_{1}+I+k}\left[\left(2 F_{0}+1\right)\left(2 J_{1}+1\right)\right]^{1 / 2} \\
\quad \times\left\{\begin{array}{ccc}
F_{0} & k & F_{1} \\
J_{1} & I & J_{0}
\end{array}\right\}\left(\alpha_{1} J_{1}\left\|Q^{(k)}\right\| \alpha_{0} J_{0}\right) .
\end{aligned}
$$

$I$ is the spin of the nucleus. The values of $J_{0}, J_{1}$ in Eqs. (14) and (15) should be changed by $F_{0}, F_{1}$. The probability $W\left(\alpha_{0} J_{0} M_{0} \hat{\epsilon} \mathbf{k}_{0} \rightarrow \alpha_{1} J_{1} M_{1}\right)$ equals to the cross-section (2) divided by the density of the flow of the radiation. Then the same expressions of the crosssection can be used for the probability, only the definition of the constant $C$ should be changed.

\section{Photoexcitation as the first step of a multistep process}

The excitation of an atom by laser or other electromagnetic radiation is often used to prepare the atom in a polarized state. Then the magnetic state of an atom is not observed, and summation over $M_{1}$ has to be performed coherently. In this case, the excitation (1) is the first step of the multistep process, while the second step is

$$
A^{*}\left(\alpha_{1} J_{1} M_{1}\right)+b(\alpha) \rightarrow A\left(\alpha_{2} J_{2} M_{2}\right)+b^{\prime}\left(\alpha^{\prime}\right) .
$$

Here $b(\alpha)$ stands for the impacting particle or electromagnetic radiation in the state $\alpha$, and $b^{\prime}\left(\alpha^{\prime}\right)$ indicates one or more ionized and emitted particles. In two-step approximation, the probability of the processes (1) and (17) can be written as coherent sum, since the projection $M_{1}$ cannot be observed [18]:

$$
\begin{aligned}
W^{t}\left(\alpha_{0} J_{0} M_{0} \hat{\epsilon} \mathbf{k}_{0} \rightarrow \alpha_{1} J_{1} \alpha \rightarrow \alpha_{2} J_{2} M_{2} \alpha^{\prime}\right) \\
=C^{\prime} \mid \sum_{M_{1}}\left\langle\alpha_{2} J_{2} M_{2} \alpha^{\prime}\left|H_{2}\right| \alpha_{1} J_{1} M_{1} \alpha\right\rangle \\
\quad \times\left.\left\langle\alpha_{1} J_{1} M_{1}\left|H_{1}\right| \alpha_{0} J_{0} M_{0}\right\rangle\right|^{2} \\
=C^{\prime} \sum_{M_{1}, M_{1}^{\prime}}\left\langle\alpha_{2} J_{2} M_{2} \alpha^{\prime}\left|H_{2}\right| \alpha_{1} J_{1} M_{1} \alpha\right\rangle \\
\quad \times\left\langle\alpha_{2} J_{2} M_{2} \alpha^{\prime}\left|H_{2}\right| \alpha_{1} J_{1} M_{1}^{\prime} \alpha\right\rangle^{*} \\
\quad \times\left\langle\alpha_{1} J_{1} M_{1}\left|H_{1}\right| \alpha_{0} J_{0} M_{0}\right\rangle \\
\quad \times\left\langle\alpha_{1} J_{1} M_{1}^{\prime}\left|H_{1}\right| \alpha_{0} J_{0} M_{0}\right\rangle^{*} .
\end{aligned}
$$

$H_{1}$ and $H_{2}$ are the operators of the interaction in the first and second processes, respectively. Then the product (11) should be changed by

$$
\begin{aligned}
& D_{\widetilde{M}_{1} M_{1}}^{J_{1}}\left(\widehat{J}_{1}\right) D_{\widetilde{M}_{1}^{\prime} M_{1}^{\prime}}^{* J_{1}^{\prime}}\left(\widehat{J}_{1}\right) \\
& =\sum_{K_{1}, N_{1}, N_{1}^{\prime}}\left[\begin{array}{ccc}
J_{1}^{\prime} & K_{1} & J_{1} \\
\widetilde{M}_{1}^{\prime} & N_{1} & \widetilde{M}_{1}
\end{array}\right] \\
& \quad \times T_{N_{1} N_{1}^{\prime}}^{* K_{1}}\left(J_{1}, J_{1}^{\prime}, M_{1}, M_{1}^{\prime} \mid \widehat{J}_{1}\right),
\end{aligned}
$$

where the tensorial operator (12) acquires a more general form

$$
\begin{aligned}
& T_{N_{1} N_{1}^{\prime}}^{K_{1}}\left(J_{1}, J_{1}^{\prime}, M_{1}, M_{1}^{\prime} \mid \widehat{J_{1}}\right) \\
& =(-1)^{J_{1}^{\prime}-M_{1}^{\prime}}\left[\frac{2 K_{1}+1}{2 J_{1}+1}\right]^{1 / 2}\left[\begin{array}{ccc}
J_{1} & J_{1}^{\prime} & K_{1} \\
M_{1} & M_{1}^{\prime} & N_{1}^{\prime}
\end{array}\right] \\
& \quad \times D_{N_{1} N_{1}^{\prime}}^{* K_{1}}\left(\widehat{J}_{1}\right) .
\end{aligned}
$$

Now the summation over $M_{1}$ and $M_{1}^{\prime}$ in Eq. (18) may be carried out. From the examination of expressions (29) in [4] and (13) of the present work, for both terms in (18) it follows that only the tensors $T_{N_{1} N_{1}^{\prime}}^{K_{1}}\left(J_{1}, J_{1}^{\prime}, M_{1}, M_{1}^{\prime} \mid \widehat{J}_{1}\right)$ depend on $M_{1}$ and $M_{1}^{\prime}$. The sum over $M_{1}, M_{1}^{\prime}$ of the product of these tensors is equal to

$$
\begin{aligned}
\sum_{M_{1}, M_{1}^{\prime}} & T_{N_{1} N}^{K_{1}}\left(J_{1}, J_{1}, M_{1}, M_{1}^{\prime} \mid \widehat{J_{1}}\right) \\
& \times T_{N_{1}^{\prime} N^{\prime}}^{* K_{1}^{\prime}}\left(J_{1}, J_{1}, M_{1}, M_{1}^{\prime} \mid \widehat{J}_{1}\right) \\
= & \frac{\sqrt{\left(2 K_{1}+1\right)\left(2 K_{1}^{\prime}+1\right)}}{2 J_{1}+1} D_{N_{1} N}^{* K_{1}}(\widehat{J}) D_{N_{1}^{\prime} N^{\prime}}^{K_{1}^{\prime}}(\widehat{J}) \\
& \times \sum_{M_{1}, M_{1}^{\prime}}(-1)^{2 J_{1}-2 M_{1}^{\prime}}\left[\begin{array}{ccc}
J_{1} & J_{1} & K_{1} \\
M_{1} & M_{1}^{\prime} & N
\end{array}\right]\left[\begin{array}{ccc}
J_{1} & J_{1} & K_{1}^{\prime} \\
M_{1} & M_{1}^{\prime} N^{\prime}
\end{array}\right] \\
= & \frac{2 K_{1}+1}{2 J_{1}+1} D_{N_{1} N}^{* K_{1}}(\widehat{J}) D_{N_{1}^{\prime} N}^{K_{1}}(\widehat{J}) \\
& \times \delta\left(K_{1}, K_{1}^{\prime}\right) \delta\left(N, N^{\prime}\right) .
\end{aligned}
$$

Then the quantization axis can be chosen along the $z$ axis of the laboratory coordinate system, and

$$
D_{N_{1} N}^{* K_{1}}(0,0,0) D_{N_{1}^{\prime} N}^{K_{1}}(0,0,0)=\delta\left(N_{1}, N\right) \delta\left(N_{1}^{\prime}, N\right) .
$$


The square root of the multiplier $\left(2 K_{1}+1\right) /\left(2 J_{1}+1\right)$ is convenient to attribute to both terms of the following expression:

$$
\begin{gathered}
W^{t}\left(\alpha_{0} J_{0} M_{0} \hat{\epsilon} \mathbf{k}_{0} \rightarrow \alpha_{1} J_{1} \alpha \rightarrow \alpha_{2} J_{2} M_{2} \alpha^{\prime}\right) \\
=\sum_{K_{1}, N_{1}} W_{K_{1} N_{1}}\left(\alpha_{0} J_{0} M_{0} \widehat{\epsilon} \mathbf{k}_{0} \rightarrow \alpha_{1} J_{1} \alpha\right) \\
\quad \times W_{K_{1} N_{1}}^{A}\left(\alpha_{1} J_{1} \alpha \rightarrow \alpha_{2} J_{2} M_{2} \alpha^{\prime}\right) .
\end{gathered}
$$

Here the sum over $M_{1}, M_{1}^{\prime}$ in Eq. (18) is changed by the sum over $K_{1}, N_{1}$, i.e. the probability of the twostep process is expanded as the sum of state multipoles [11]. Then the expressions for the photoexcitation probability and that of the second process slightly change. For the excitation probability, it is

$$
\begin{aligned}
& \frac{\mathrm{d} W_{K_{1} N_{1}}\left(\alpha_{0} J_{0} M_{0} \hat{\epsilon} \mathbf{k}_{0} \rightarrow \alpha_{1} J_{1}\right)}{\mathrm{d} \Omega} \\
& =\frac{C}{\left[2 J_{1}+1\right]^{1 / 2}} \sum_{K_{0}, K_{r}, k, k^{\prime}} B^{r}\left(K_{0}, K_{r}, K_{1}, k, k^{\prime}\right) \\
& \times \sum_{N_{0}, N_{r}, q}\left[\begin{array}{lll}
K_{0} & K_{r} & K_{1} \\
N_{0} & N_{r} & N_{1}
\end{array}\right] \\
& \times T_{N_{0}}^{* K_{0}}\left(J_{0}, J_{0}, M_{0} \mid \widehat{J}_{0}\right) T_{N_{r}}^{* K_{r}}\left(k, k^{\prime}, q \mid \widehat{k}_{0}\right), \\
& W_{00}\left(\alpha_{0} J_{0} k \rightarrow \alpha_{1} J_{1}\right) \\
& =\frac{C}{\left(2 J_{0}+1\right)(2 k+1)\left[2 J_{1}+1\right]^{1 / 2}} \\
& \times\left|\left\langle\alpha_{1} J_{1}\left\|Q^{(k)}\right\| \alpha_{0} J_{0}\right\rangle\right|^{2} \text {. }
\end{aligned}
$$

The expression for the second term in Eq. (22) depends on the second step process. In the case of the Auger decay, it is presented by Kupliauskiene and Tutlys (see Eq. (4) in [19]).

The proposed method is easy to generalize for multistep process when intermediate states are not observed. In the case of a three-step process where the fluorescence radiation of the doubly charged ion formed after the photoionization of an atom and the Auger decay of a singly charged ion is registered, the summation over intermediate states gives

$$
\begin{gathered}
\sum_{M_{1}, M_{1}^{\prime}, M_{2}, M_{2}^{\prime}} W^{1}\left(J_{0} M_{0} \mathbf{k}_{1} \rightarrow J_{1} M_{1} M_{1}^{\prime} \mathbf{p}_{1} m_{1}\right) \\
\times W^{2}\left(J_{1} M_{1} M_{1}^{\prime} \mathbf{p}_{1} m_{1} \rightarrow J_{2} M_{2} M_{2}^{\prime} \mathbf{p}_{2} m_{2}\right) \\
\times W^{3}\left(J_{2} M_{2} M_{2}^{\prime} \mathbf{p}_{2} m_{2} \rightarrow J_{3} M_{3} \mathbf{k}_{2}\right)
\end{gathered}
$$

$$
\begin{aligned}
= & \sum_{K_{1}, N_{1}, K_{2}, N_{2}} W_{K_{1} N_{1}}^{1}\left(J_{0} M_{0} \mathbf{k}_{1} \rightarrow J_{1} \mathbf{p}_{1} m_{1}\right) \\
& \times \frac{2 K_{1}+1}{2 J_{1}+1} W_{K_{1} N_{1} K_{2} N_{2}}^{2}\left(J_{1} \mathbf{p}_{1} m_{1} \rightarrow J_{2} \mathbf{p}_{2} m_{2}\right) \\
& \times \frac{2 K_{2}+1}{2 J_{2}+1} W_{K_{2} N_{2}}^{3}\left(J_{2} \mathbf{p}_{2} m_{2} \rightarrow J_{3} M_{3} \mathbf{k}_{2}\right) .(25)
\end{aligned}
$$

The square root of each multiplier $(2 K+1) /(2 J+1)$ in Eq. (25) is also convenient to attribute to both terms standing side by side.

\section{Practical applications}

The expressions (13) and (23) represent the general case of the excitation of polarized atoms by polarized radiation. Two cases are chosen to demonstrate their practical application. The first case is the excitation of randomly oriented atoms by the circularly and linearly polarized dipole radiation. The second one is the excitation of polarized atoms by unpolarized radiation. Since usually the photoexcitation is used to prepare an atom in a polarized state, the practical application of the expression (13) is limited. Therefore, the abovementioned expressions will be obtained for the excitation probability in the first step process characterized by Eq. (23).

\subsection{Photoexcitation of unpolarized atoms}

To obtain the expression for the probability describing the excitation of an unpolarized atom by polarized dipole radiation, one needs to average the probability (23) over the initial states of an atom. The use of the expression (A1) from [15]

$$
\sum_{M_{0}} T_{N_{0}}^{K_{0}}\left(J_{0}, J_{0}, M_{0} \mid \widehat{J}_{0}\right)=\delta(K, 0) \delta(N, 0)
$$

in Eq. (23) enables us to write

$$
\begin{aligned}
& \frac{\mathrm{d} W_{K N}\left(\alpha_{0} J_{0} \hat{\epsilon} \mathbf{k}_{0} \rightarrow \alpha_{1} J_{1}\right)}{\mathrm{d} \Omega} \\
& =\frac{4 \pi C}{\left(2 J_{0}+1\right) \sqrt{3\left(2 J_{1}+1\right)}} \\
& \quad \times \sum_{K, q}(-1)^{1-q} B^{r}(0, K, K, 1,1) \\
& \quad \times\left[\begin{array}{ccc}
1 & 1 & K \\
q-q & 0
\end{array}\right] Y_{K N}(\theta, \phi) .
\end{aligned}
$$


Here $\theta, \phi$ are polar and azimuthal angles between the direction of incoming radiation for the circularly polarized and unpolarized light, or its electrical vector $\mathbf{E}$ in the case of linearly polarized light, and laboratory $z$ axis. When these directions coincide with the laboratory $z$ axis, by using

$$
\begin{aligned}
& Y_{K N}^{*}(0,0)=\left[\frac{2 K+1}{4 \pi}\right]^{1 / 2} \delta(N, 0), \\
& Y_{K 0}(\theta, \phi)=\left[\frac{2 K+1}{4 \pi}\right]^{1 / 2} P_{K}(\cos \theta),
\end{aligned}
$$

the final expression may be written in the form

$$
\begin{aligned}
W_{K 0}\left(\alpha_{0} J_{0} q \rightarrow \alpha_{1} J_{1} M_{1}\right) & \\
= & (-1)^{1-q} \frac{C}{2 J_{0}+1}\left[\frac{2 K+1}{3\left(2 J_{1}+1\right)}\right]^{1 / 2} \\
& \times B^{r}(0, K, K, 1,1)\left[\begin{array}{ccc}
1 & 1 & K \\
q & -q & 0
\end{array}\right], \\
B^{r}(0, K, K, 1,1) & \\
= & \left(\alpha_{1} J_{1}\left\|Q^{(1)}\right\| \alpha_{0} J_{0}\right)^{2}(-1)^{1+K+J_{1}+J_{0}} \\
& \times\left[\frac{\left(2 J_{1}+1\right) 3}{2 K+1}\right]^{1 / 2}\left\{\begin{array}{ccc}
1 & 1 & K \\
J_{1} & J_{1} & J_{0}
\end{array}\right\},
\end{aligned}
$$

and $P_{K}(\cos \theta)$ is the Legendre polynomial [16].

In the case of circularly polarized electrical dipole radiation, $k=1, q= \pm 1, K=0,1,2$, the excited atom is oriented along the laboratory $z$ axis if $J_{1} \geq 1 / 2$ because of the condition $K \leq 2 J_{1}$. The ranks $K=1$ and $K=2$ describe the orientation and alignment [11], respectively. For the linearly polarized light, $q=0$ and $K=0,2$ because the Clebsch-Gordan coefficient in Eq. (30) is equal to zero when $q=0$ and $2+K$ is odd [10]. The unpolarized light is described by the sum of equal parts of the right- and left-hand circularly polarized radiation. Then the terms of the sum with $K=1$ cancel, and only the terms with $K=0,2$ bring the contribution like in the case of linearly polarized light, but one must have in mind that $q=1$. This means that the excited atom is created in the aligned state even if excitated by unpolarized radiation. For the description of orientation and alignment, the parameters $A_{1}$ and $A_{2}$, respectively, were introduced [12]. In the case of ex- citation by electrical dipole radiation, their expressions are

$$
\begin{aligned}
A_{1}=\frac{W_{10}}{W_{00}}= & 3 \sqrt{2 J_{1}+1}(-1)^{J_{1}+J_{0}+1-q} \\
\times & {\left[\begin{array}{ccc}
1 & 1 & 1 \\
q & -q & 0
\end{array}\right]\left\{\begin{array}{ccc}
1 & 1 & 1 \\
J_{1} & J_{1} & J_{0}
\end{array}\right\}, } \\
A_{2}=\frac{W_{20}}{W_{00}}= & 3 \sqrt{2 J_{1}+1}(-1)^{J_{1}+J_{0}-q} \\
& \times\left[\begin{array}{ccc}
1 & 1 & 2 \\
q & -q & 0
\end{array}\right]\left\{\begin{array}{ccc}
1 & 1 & 2 \\
J_{1} & J_{1} & J_{0}
\end{array}\right\} .
\end{aligned}
$$

For the linearly polarized dipole radiation, $A_{1}=0$, and

$$
A_{2}^{\mathrm{L}}=\sqrt{6\left(2 J_{1}+1\right)}(-1)^{J_{1}+J_{0}}\left\{\begin{array}{ccc}
1 & 1 & 2 \\
J_{1} & J_{1} & J_{0}
\end{array}\right\} .
$$

In the case of circularly polarized dipole radiation, $A_{2}$ coincides with that of unpolarized radiation:

$$
A_{2}=\left[\frac{3\left(2 J_{1}+1\right)}{2}\right]^{1 / 2}(-1)^{J_{1}+J_{0}-1}\left\{\begin{array}{ccc}
1 & 1 & 2 \\
J_{1} & J_{1} & J_{0}
\end{array}\right\}
$$

and

$$
A_{1}=3\left[\frac{2 J_{1}+1}{2}\right]^{1 / 2}(-1)^{J_{1}+J_{0}}\left\{\begin{array}{ccc}
1 & 1 & 1 \\
J_{1} & J_{1} & J_{0}
\end{array}\right\} .
$$

The calculated parameters describing the orientation $A_{1}$ (36) and alignment $A_{2}$ (34)-(35) of atoms excited by the electrical dipole radiation are presented in Table 1. The orientation and alignment of the resonance state in the case of photoexcitation are welldetermined and both are model-independent because they do not depend on the values of the reduced matrix elements (see Eqs. (32)-(36)). Therefore, they can be used for the investigation of any atom in any experiment. The values of these coefficients can differ from those obtained by using the density matrix formalism by $\sqrt{2 J_{1}+1}$ (e. g., [9]) because of our definition (25) where $[(2 K+1) /(2 J+1)]^{1 / 2}$ is attributed to each term standing side by side.

The data from Table 1 show that all atoms excited to the states $J_{1} \geq 1 / 2$ by electric dipole radiation are oriented $\left(J_{1} \geq 1 / 2\right)$ and aligned $\left(J_{1} \geq 1\right)$. This polarization was observed in the second step process products. The transferred spin polarization of the resonantly excited $3 d_{J}^{-1} 5 p$ Auger electrons of krypton was registered [20] to be $80 \%$. 
Table 1. Parameters describing orientation $A_{1}$ and alignment $A_{2}$ in the case of right circularly polarized dipole radiation and alignment $A_{2}^{\mathrm{L}}$ for the linearly polarized radiation.

\begin{tabular}{cccccccccc}
\hline$J_{0}$ & $J_{1}$ & $A_{1}$ & $A_{2}$ & $A_{2}^{\mathrm{L}}$ & $J_{0}$ & $J_{1}$ & \multicolumn{1}{c}{$A_{1}$} & \multicolumn{1}{c}{$A_{2}$} & \multicolumn{1}{c}{$A_{2}^{\mathrm{L}}$} \\
\hline 0 & 1 & 1.2247 & 0.7071 & -1.4142 & $5 / 2$ & $3 / 2$ & -0.6708 & 0.1 & \multicolumn{1}{c}{-0.2} \\
$1 / 2$ & $1 / 2$ & 1 & 0 & 0 & & $5 / 2$ & 0.2928 & -0.4276 & \multicolumn{1}{c}{0.8552} \\
& $3 / 2$ & 1.1180 & 0.5 & -1 & & $7 / 2$ & 0.9820 & 0.3873 & -0.6546 \\
1 & 0 & 0 & 0 & 0 & 3 & 2 & -0.7071 & 0.1195 & -0.2390 \\
& 1 & 0.6124 & -0.3536 & 0.7071 & & 3 & 0.25 & -0.4330 & 0.8660 \\
& 2 & 1.0607 & 0.4183 & -0.8367 & & 4 & 0.9682 & 0.3134 & -0.6268 \\
$3 / 2$ & $1 / 2$ & -0.5 & 0 & 0 & $7 / 2$ & $5 / 2$ & -0.7319 & 0.1336 & -0.2673 \\
& $3 / 2$ & 0.4472 & -0.4 & 0.8 & & $7 / 2$ & 0.2182 & -0.4364 & 0.8729 \\
& $5 / 2$ & 1.0247 & 0.3742 & -0.7483 & & $9 / 2$ & 0.9574 & 0.3028 & -0.6055 \\
2 & 1 & -0.6124 & 0.0707 & -0.1414 & 4 & 3 & -0.75 & 0.1443 & -0.2887 \\
& 2 & 0.3536 & -0.4183 & 0.8367 & & 4 & 0.1936 & -0.4387 & 0.8775 \\
& 3 & 1 & 0.3464 & -0.6928 & & 5 & 0.9487 & 0.2944 & -0.5889 \\
\hline
\end{tabular}

\subsection{Photoexcitation of polarized atoms}

In the second case of the excitation of polarized atoms by unpolarized radiation, one needs to take into account the earlier mentioned description of unpolarized light and the condition that $K=0,2$, as well as that the laboratory $z$ axis is aligned along the direction of incoming radiation. Then $N_{r}=0$ due to the relation (28), and the contribution of terms of only the same multipolarity Eq. (22) acquires a simpler form:

$$
\begin{aligned}
W_{K_{1} N_{1}}\left(\alpha_{0} J_{0} M_{0} q \rightarrow \alpha_{1} J_{1}\right) \\
=C\left[\frac{4 \pi}{\left(2 J_{0}+1\right)(2 k+1)\left(2 J_{1}+1\right)}\right]^{1 / 2} \\
\quad \times(-1)^{J_{0}-M_{0}+1-q} \sum_{K_{0}, K_{r}}\left[2 K_{r}+1\right]^{1 / 2} \\
\quad \times B^{r}\left(K_{0}, K_{r}, K_{1}, 1,1\right)\left[\begin{array}{ccc}
K_{0} & K_{r} & K_{1} \\
N_{1} & 0 & N_{1}
\end{array}\right] \\
\quad \times\left[\begin{array}{ccc}
J_{0} & J_{0} & K_{0} \\
M_{0}-M_{0} & 0
\end{array}\right]\left[\begin{array}{ccc}
k & k & K_{r} \\
q-q & 0
\end{array}\right] Y_{K_{0} N_{1}}(\theta, \phi) .
\end{aligned}
$$

The angles $\theta$ and $\phi$ of the total angular momentum $J_{0}$ are measured from the direction of incoming radiation.

In the electrical dipole approximation, $K_{r}=0,2$, Eq. (37) can be written as

$$
\begin{aligned}
& W_{K_{1} N_{1}}\left(\alpha_{0} J_{0} M_{0} \rightarrow \alpha_{1} J_{1}\right) \\
& =W_{00}\left(\alpha_{0} J_{0} \rightarrow \alpha_{1} J_{1}\right) \\
& \quad \times\left[1+A_{2}\left(J_{0} M_{0}, K_{1} N_{1}, \theta, \phi\right)\right],
\end{aligned}
$$

$$
\begin{aligned}
& A_{2}\left(J_{0} M_{0}, K_{1} N_{1}, \theta, \phi\right) \\
& =\sum_{K_{0}}\left[\frac{4 \pi}{2 K_{0}+1}\right]^{1 / 2} \\
& \times Y_{K_{0} N_{1}}(\theta, \phi) \beta\left(K_{0}, 2, K_{1}\right)\left[\begin{array}{lll}
K_{0} & 2 & K_{1} \\
N_{1} & 0 & N_{1}
\end{array}\right], \\
& \beta\left(K_{0}, 2, K_{1}\right) \\
& =(-1)^{J_{0}-M_{0}+1-q} 3\left(2 J_{0}+1\right) \\
& \times\left[\left(2 J_{1}+1\right)\left(2 K_{1}+1\right)\left(2 K_{0}+1\right)\left(2 K_{r}+1\right)\right]^{1 / 2} \\
& \times\left[\begin{array}{ccc}
1 & 1 & K_{r} \\
q-q & 0
\end{array}\right]\left[\begin{array}{ccc}
J_{0} & J_{0} & K_{0} \\
M_{0}-M_{0} & 0
\end{array}\right]\left\{\begin{array}{ccc}
J_{0} & K_{0} & J_{0} \\
1 & 2 & 1 \\
J_{1} & K_{1} & J_{1}
\end{array}\right\} .
\end{aligned}
$$

Parameter $A_{2}\left(J_{0} M_{0}, K_{1} N_{1}, \theta, \phi\right)$ describes the alignment of the excited atom that was created by the photoexcitation of a polarized atom. It depends on the orientation of the atom in its initial state, but is independent of the value of the reduced matrix element. The values of $A_{2}\left(J_{0} M_{0}, K_{1} N_{1}\right)$ can be calculated if the orientation of the atom is known. In the case of $J_{0}$ aligned along the $z$ axis that coincides with the direction of exciting radiation, the expression for $A_{2}\left(J_{0} M_{0}, K_{1} N_{1}\right)$ is simple, because $M_{0}=J_{0}, K_{0}+K_{1}=$ even, and $N_{1}=0$ :

$$
\begin{aligned}
A_{2}\left(J_{0}\right. & \left.J_{0}, K_{1} 0,0,0\right) \\
= & 3\left(2 J_{0}+1\right) \\
& \times \sum_{K_{0} \leq 2 J_{0}}\left[5\left(2 J_{1}+1\right)\left(2 K_{1}+1\right)\left(2 K_{0}+1\right) / 6\right]^{1 / 2} \\
& \times\left[\begin{array}{ccc}
K_{0} & 2 & K_{1} \\
0 & 0 & 0
\end{array}\right]\left[\begin{array}{ccc}
J_{0} & J_{0} & K_{0} \\
J_{0} & -J_{0} & 0
\end{array}\right]\left\{\begin{array}{ccc}
J_{0} & K_{0} & J_{0} \\
1 & 2 & 1 \\
J_{1} & K_{1} & J_{1}
\end{array}\right\} \cdot(41)
\end{aligned}
$$


Table 2. Parameters describing the alignment $A_{2}\left(J_{0}, J_{0}=M_{0}, K_{1} 0,0,0\right)$ in the case of the photoexcitation of atoms polarized along the direction of incoming unpolarized radiation $\left(J_{0} \leq 3, J_{1} \leq 3\right)$.

\begin{tabular}{|c|c|c|c|c|c|c|c|}
\hline \multirow[b]{2}{*}{$J_{0}$} & \multirow[b]{2}{*}{$J_{1}$} & \multicolumn{6}{|c|}{$K_{1}$} \\
\hline & & 1 & 2 & 3 & 4 & 5 & 6 \\
\hline 0 & 1 & & 1.58811 & & & & \\
\hline \multirow[t]{2}{*}{$1 / 2$} & $1 / 2$ & -1.1547 & & & & & \\
\hline & $3 / 2$ & 0.2582 & & 1.7748 & & & \\
\hline \multirow[t]{2}{*}{1} & 1 & & -1.5811 & & & & \\
\hline & 2 & & 1.0690 & & 1.4432 & & \\
\hline \multirow[t]{3}{*}{$3 / 2$} & $1 / 2$ & 0.8660 & & & & & \\
\hline & $3 / 2$ & -1.2394 & & -1.4199 & & & \\
\hline & $5 / 2$ & 0.4564 & & 1.6424 & & 1.0235 & \\
\hline \multirow[t]{3}{*}{2} & 1 & & 0.7906 & & & & \\
\hline & 2 & & -2.0045 & & -1.0690 & & \\
\hline & 3 & & 1.1066 & & 1.7665 & & 0.6725 \\
\hline \multirow[t]{2}{*}{$5 / 2$} & $3 / 2$ & 1.1619 & & 0.5916 & & & \\
\hline & $5 / 2$ & -1.5937 & & -2.2082 & & -0.7311 & \\
\hline \multirow[t]{2}{*}{3} & 2 & & 1.3363 & & 0.4009 & & \\
\hline & 3 & & -2.4206 & & -1.9988 & & -0.4707 \\
\hline
\end{tabular}

In Eq. (41), $K_{1} \leq 2 J_{1}$.

The calculated values for $A_{2}\left(J_{0} J_{0}, K_{1} 0,0,0\right)$ in the case of $J_{0} \leq 3$ and $J_{1} \leq 3$ are presented in Table 2 . They are also model-independent and can be used for the investigation of any atom in any experiment.

\section{Concluding remarks}

To summarize, a general expression for the excitation probability of polarized atoms by polarized radiation was obtained. The integration over the orbital and spin variables was performed by using the graphical technique of angular momentum. The approach is based on the ordinary atomic theory and is an alternative to the density matrix formalism in the case of pure states. Since usually the photoexcitation is used to prepare an atom in a polarized state, the general expression was also obtained for the probability of excitation as the first step process. The generalization of the obtained expressions in the case of a multistep process when intermediate states are not observed is presented. Two cases are chosen to demonstrate the practical application. The first case is the excitation of randomly oriented atoms by the unpolarized and by the circularly and linearly polarized dipole radiation. The second one is the excitation of polarized atoms by unpolarized radiation. The parameters describing the orientation and alignment in the case of photoexcitation are well-determined and both are model-independent because they do not depend on the values of the reduced matrix elements. Therefore, the calculated values of these parameters in the present work can be used for the investigation of any atom in any experiment.

\section{References}

[1] Ph. Golecki and H. Klar, (e, 2e) from laser-excited atoms with spin-polarized electrons, J. Phys. B 32, $1647-1656$ (1999).

[2] H. Aksela, Resonant Auger spectroscopy of atoms and molecules, J. Electron Spectrosc. 72, 235-242 (1995).

[3] K. Ueda, Y. Shimizu, H. Chiba, Y. Sato, M. Kitajima, H. Tanaka, and N.M. Kabachnik, Experimental determination of Auger-decay amplitudes from the angular correlations in Auger cascades following the $2 p \rightarrow 4 s$ photoexcitation of Ar, Phys. Rev. Lett. 83, 5463-5466 (1999).

[4] B. Langer, N. Berrah, A. Farhat, M. Humphrey, D. Cubaynes, A. Menzel, and U. Becker, Angular distributions of resonant and non-resonant Auger electrons as a test case for the validity of spectator model: The argon $L_{2} M M$ case, J. Phys. B 30, 4255-4266 (1977).

[5] P. O'Keeffe, S. Aloise, M. Meyer, and A.N. GrumGrzhimailo, Circular polarization of ion fluorescence completing the analysis of resonant $\mathrm{Xe}^{*} 4 d_{5 / 2}^{-1} 6 p$ Auger decay, Phys. Rev. Lett. 90, 023002(4) (2003).

[6] S.C. McFarlane, The polarization of characteristic X-radiation excited by electron impact, J. Phys. B 5, 1906-1915 (1972).

[7] A.N. Grum-Grzhimailo and N.M. Kabachnik, Linear magnetic dichroism in fluorescence spectra, Phys. Lett. A 264, 192-197 (1999).

[8] N.M. Kabachnik, I.P. Sazhina, and K. Ueda, Angular distribution of Auger electrons and fluorescence in cascades and resonantly enhanced transitions, J. Phys. B 32, 1769-1781 (1999). 
[9] V.V. Balashov, A.N. Grum-Grzhimailo, and N.M. Kabachnik, Angular distribution of autoionization and Auger electrons ejected by electron impact from laser excited and ionized atoms, J. Phys. B 30, 1269-1281 (1997).

[10] A.P. Jucys and A.A. Bandzaitis, Theory of Angular Momentum in Quantum Mechanics (Mintis, Vilnius, 1965) [in Russian].

[11] K. Blum, Density Matrix Theory and Applications, 2nd edn. (Plenum, New York, 1996).

[12] V.V. Balashov, A.N. Grum-Grzhimailo, and N.M. Kabachnik, Polarization and Correlation Phenomena in Atomic Collisions. A Practical Theory Course (Kluwer, New York, 2000).

[13] A. Kupliauskienè, N. Rakštikas, and V. Tutlys, General expression of the photoionization cross-section of an atom in polarized $L S$ state, Lithuanian J. Phys. 40, 311-320 (2000).

[14] A. Kupliauskienè, N. Rakštikas, and V. Tutlys, Polarization studies in the photoionization of atoms using a graphical technique, J. Phys. B 34, 1783-1803 (2001).
[15] A. Kupliauskiené and V. Tutlys, Application of graphical technique for Auger decay following photoionization of atoms, Physica Scripta 67, 290-300 (2003).

[16] D.A. Varshalovich, A.N. Moskalev, and V.K. Khersonskii, Quantum Theory of Angular Momentum (World Scientific, Singapore, 1988).

[17] B. Krassing, J.-C. Bilbeux, R.W. Dunford, D.S. Gemmell, S. Hasegawa, E.P. Hanter, S.H. Southworth, L. Young, L.A. LaJohn, and R.H. Prat, Nondipole asymmetries of $\mathrm{Kr} 1 s$ photoelectrons, Phys. Rev. A 67, 022707 (2003).

[18] H. Klar, Polarization of fluorescence radiation following atomic photoionization, J. Phys. B 13, 2037-2049 (1980).

[19] A. Kupliauskienė and V. Tutlys, Auger decay probability following photoionization of atoms, Lithuanian J. Phys. 43, 27-34 (2003).

[20] B. Lohmann, U, Hergenhahn, and N.M. Kabachnik, Spin polarization of Auger electrons from noble gases after photoionization with circularly polarized light, J. Phys. B 26, 3327-3338 (1993).

\title{
POLIARIZUOTO ATOMO SUŽADINIMAS POLIARIZUOTA SPINDULIUOTE
}

\author{
A. Kupliauskienè \\ VU Teorines fizikos ir astronomijos institutas, Vilnius, Lietuva
}

\section{Santrauka}

Derinamo dažnio lazerio ir sinchrotroninè spinduliuotès atveria galimybę ne tiktai jonizuoti atomus, atplěšiant elektronus iš pageidaujamo sluoksnio, bet ir juos sužadinti i pageidaujamą būseną. Šitaip paruošiami poliarizuoti atomai, kurie toliau naudojami poliarizacijos reiškiniams jonizuojant atomus elektronais ir fotonais tirti. Norint vieningai teoriškai aprašyti atomo sužadinimo ir po jo einančius jonizacijos vyksmus bei tirti, kaip antraji vyksmą apibūdinantys dydžiai priklauso nuo pirmojo, reikalingos kuo bendresnès tokius vyksmus aprašančios formulès. Panaudojant judèjimo kiekio momento grafini vaiz- davimą ir atomo teorijos metodus, surasta poliarizuoto atomo sužadinimo poliarizuota spinduliuote tikimybės išraiška. Grynųju būsenų atveju panaudotasis metodas yra alternatyvus iki šiol taikytam tankio matricos formalizmui. Surastoji formule taip pat aprašo sužadinto atomo poliarizaciją. Ji panaudota nepoliarizuoto atomo sužadinimo poliarizuota spinduliuote ir poliarizuoto atomo sužadinimo nepoliarizuota spinduliuote tikimybès išraiškoms rasti, laikant, kad sužadinimas tèra pirmoji tolimesnio vyksmo stadija. Taip pat parodyta, kokius pakeitimus reikia padaryti, kad formulès būtų pritaikytos daugiapakopiams vyksmams aprašyti. 